Chicago, in 1965, demonstrated a reduction in the seizure threshold following adrenalectomy and in both intact and adrenalectomized young rats, following ACTH. Measurements of brain and plasma electrolytes showed that the reduced seizure threshold was correlated with an increase in the concentration of intracellular sodium in the brain. The ACTH effects on seizure threshold and brain sodium were independent of the adrenal. Animals with seizure thresholds experimentally lowered by methods other than adrenalectomy might demonstrate the anticonvulsant effect of ACTH observed clinically (Wasserman MJ, Belton NR, Millichap JG. Effect of corticotropin (ACTH) on experimental seizures. Adrenal independence and relation to intracellular brain sodium. Neurology Dec $1965 ; 15: 1136-1144)$.

\title{
NOCTURNAL FAMILIAL FRONTAL LOBE EPILEPSY
}

A clinical and video-polysomnographic (VPS) study in 40 patients complaining of repeated abnormal nocturnal motor and/or behavioral phenomena, from 30 unrelated Italian families, is reported from the Sleep Disorders Centre and the Department of Biology and Genetics for Medical Sciences, University of Milan, Italy. A diagnosis of autosomal dominant nocturnal frontal lobe epilepsy (ADFLE) was strongly suggested by electroclinical manifestations, which included nocturnal enuresis, violent behavior, and other features seen also in 'typical' parasomnias. The VPS recordings confirmed sudden awakenings and dystonic/dyskinetic movements in $42 \%$, complex behaviors in $13 \%$, and violent behavior in $5 \%$. Ictal epileptiform abnormalities and rhythmic slow activity in frontal areas were recorded on the EEG in $32 \%$ and $47 \%$ of patients, respectively. A correct diagnosis of epilepsy had been made previously in only $18 \%$, and $>80 \%$ had been misdiagnosed as parasomnia. Seizures were controlled by antiepileptic drugs in $73 \%$. Pedigree analysis on 28 of the families showed an autosomal dominant transmission with reduced penetrance $(81 \%)$. Nucleotide sequence analysis failed to uncover the missense mutation in the CHRNA4 gene previously reported, but did confirm two other base substitutions and supported the locus heterogeneity of ADFLE. (Oldani A, Zucconi M, Asselta R, et al. Autosomal dominant nocturnal frontal lobe epilepsy. A video-polysomnoghraphic and genetic appraisal of 40 patients and delineation of the epileptic syndrome. Brain Feb 1998;121:205-223). (Respond: Alessandro Oldani MD, Sleep Disorders Centre, IRCCS H. San Raffaele, via Prinetti 29, 20127 Milano, Italy).

COMMENT. A report of electroclinical patterns in 33 patients with autosomal dominant frontal lobe epilepsy (ADFLE), including video-polysomnographic monitoring in 12, was published by these authors in 1996 (see Progress in Pediatric Neurology III, PNB Publ, 1997, pp87-88). The present report includes further patients and a genetic analysis of the syndrome. ADFLE is often misdiagnosed as nightmares, night terrors, or somnambulism. EEGs are frequently non-specific, and video-polysomnographic monitoring is important in the differentiation of ADFLE from parasomnias. A trial of antiepileptic drugs, carbamazepine or clonazepam, is warranted when the diagnosis is suspected, particularly in patients with diurnal symptoms.

SPECT studies in ADFLE. Frontal lobe foci were unilateral and in variable locations in two patients with ADFLE reported from the Department of Neurology, Austin and Repatriation Medical Centre, Heidelberg (Melbourne), Victoria 3084, Australia (Hayman M, et al. Neurology 1997;49:969-975). One case showed a left frontal onset corroborated by congruent focal hypometabolism on intericatal PET and focal hyperperfusion on ictal SPECT. Another case studied with ictal SPECT showed a right parasagittal, midfrontal focus. 